\title{
Heat shock protein gp96 and NAD(P)H oxidase 4 play key roles in Toll-like receptor 4-activated apoptosis during renal ischemia/reperfusion injury
}

\author{
S Ben Mkaddem ${ }^{1}$, E Pedruzzi ${ }^{1}$, C Werts ${ }^{2}$, N Coant ${ }^{1}$, M Bens ${ }^{1}$, F Cluzeaud ${ }^{1}$, JM Goujon ${ }^{3}$, E Ogier-Denis ${ }^{1}$ and A Vandewalle ${ }^{\star, 1}$
}

Ischemia/reperfusion injury (IRI) causes inflammation and cell injury as a result of activating innate immune signaling. Toll-like receptor 4 (TLR4) has a key role in mediating kidney damages during IRI, but the downstream signaling pathway(s) stimulating apoptosis remains debated. In this study we show that TLR4 mediates MyD88-dependent activation of TNF receptor-associated factor 2, apoptosis signal-regulating kinase 1 (ASK1), and Jun N-terminal kinase (JNK) and p38 MAP kinases in ischemicreperfused kidneys and posthypoxic renal tubule epithelial cells (RTECs). Hypoxia stimulated the expression of the endoplasmic-resident gp96, which co-immunoprecipitated TLR4, whereas silencing gp96 mRNA expression impaired hypoxiainduced apoptosis in TLR4-expressing RTECs. NAD(P)H oxidase 4 (NOX4) was shown to interact with TLR4 and to be required in lipopolysaccharide-induced production of reactive oxygen species (ROS). IRI stimulated the expression of a 28-kDa NOX4 spliced isoform abundantly expressed in wild-type RTECs, which co-immunoprecipitated with TLR4, but not with gp96 in TLR4deficient RTECs. Silencing NOX4 mRNA expression impaired hypoxia-induced activation of ASK1 and both JNK and p38, leading to the inhibition of ROS production and apoptosis in posthypoxic TLR4-expressing RTECs. These findings show that, concomitantly to the activation of $\mathrm{p38}$, the gp96/TLR4 interaction is required for activation of ASK1/JNK signaling in posthypoxic mouse RTECs, and that the 28-kDa NOX4 has a key role in TLR4-mediated apoptosis during renal IRI.

Cell Death and Differentiation (2010) 17, 1474-1485; doi:10.1038/cdd.2010.26; published online 12 March 2010

Renal ischemia/reperfusion injury (IRI) is one of the most common causes of acute renal failure, which is associated with increased morbidity and mortality. ${ }^{1}$ It is also an unavoidable event during transplantation, and may compromise subsequent renal graft function. ${ }^{2}$ IRI induces inflammatory response and variable tissue damage ${ }^{1}$ resulting from the capacity of endogenous ligands, known as damage-associated molecular patterns (DAMPs) that are secreted or released from hypoxic or necrotic cells to activate immune signaling pathways by common pattern recognition receptors. Among DAMPs, the high-mobility group box 1 (HMGB1), heat shock proteins (Hsps), and the matrix components biglycan and heparan sulfate were shown to activate both Toll-like receptors 2 (TLR2) and 4 (TLR4). ${ }^{3}$ The role of TLR4 in the inflammation caused by IRI has been established in hepatic IRI, cardiac IRI, and hemorrhagic shock. ${ }^{4-6}$ TLR4 and TLR2 also mediate inflammatory responses and kidney damage during IRI: mice deficient for TLR2, TLR4, or the adaptor protein MyD88 (myeloid differentiation factor-88) withstand IRI better than wild-type mice..$^{7-9}$ Renal tubule epithelial cells
(RTECs) constitutively express TLR2 and TLR4, the expressions of both of which are increased in ischemic RTECs, ${ }^{10,11}$ produce cytokines, and undergo apoptosis after ischemia. ${ }^{7,9}$ However, less is known about the TLR4-mediated downstream pro-apoptotic pathway(s) activated during IRI. The endoplasmic reticulum (ER) resident gp96, homologue to cytosolic Hsp90, is a master chaperone for most TLRs. ${ }^{12} \mathrm{We}$ have shown that gp96 interacts with the protein phosphatase 5 (PP5) to control selective TLR2-mediated activation of the mitogen-activated protein kinase (MAPK) $\mathrm{ERK}_{1 / 2}$, but not of JNK. ${ }^{13}$ The question therefore arises as to whether gp96, shown to interact with TLR4 in intestinal epithelial cells, ${ }^{14}$ is required for TLR4-activated apoptosis during renal IRI.

The production of reactive oxygen species (ROS) by hypoxic cells has an important role in the induction of apoptosis. ROS-induced cell injury has been attributed in part to the activation of MAPK. ${ }^{15}$ The serine/threonine MAP kinase apoptosis signal-regulating kinase 1 (ASK1) has a pivotal role in cytokine- and stress-induced apoptosis. ${ }^{16,17}$ In response to ROS stimulation, the negative redox sensor

${ }^{1}$ INSERM, U773, Centre de Recherche Biomédicale Bichat-Beaujon CRB3, Université Paris 7 Denis Diderot, site Bichat, BP 416, Paris F-75018, France; ${ }^{2}$ Institut Pasteur, G5 Biologie et Génétique des Parois Bactériennes, Groupe INSERM Avenir, Paris Cedex 15 75724, France and ${ }^{3}$ Service d'Anatomie et Cytologie Pathologiques, Centre Hospitalier Universitaire de Poitiers, Université de Poitiers, Poitiers F-86021, France

*Corresponding author: A Vandewalle, Faculté de Médecine Xavier Bichat, INSERM, U773, Centre de Recherche Biomédicale Bichat-Beaujon, CRB3, UFR de Médecine Xavier Bichat, BP 416, 16 rue Henri Huchard, Paris Cedex 18 F-75870, France. Tel: + 331 57277550; Fax: + 331 57277531;

E-mail: alain.vandewalle@ inserm.fr

Keywords: kidney; gp96; Toll-like receptor 4; NAD(P)H oxidase 4; apoptosis

Abbreviations: ASK1, apoptosis signal-regulating kinase 1; DAMP, damage-associated molecular pattern; DPI, diphenyleneiodonium chloride; HMGB1, high-mobility group box 1; Hsps, heat shock proteins; JNK, Jun N-terminal kinase; LPS, lipopolysaccharide; MAPK, mitogen-activated protein kinase; MyD88, myeloid differentiation factor-88; NOX, NAD(P)H oxidase; PP5, protein phosphatase 5; ROS, reactive oxygen species; RTEC, renal tubule epithelial cell; siRNA, small-interfering RNA; TRAF, TNF receptor-associated factor; UPR, unfolded protein response

Received 12.10.09; revised 05.2.10; accepted 08.2.10; Edited by H Ichijo; published online 12.3.10 
thioredoxin dissociates from the endogenous inactive ASK1 complex, and then forms an active high-molecular-mass complex after the recruitment of TNF receptor-associated factors 2 (TRAF2) and 6 (TRAF6), and the downstream activation of Jun $\mathrm{N}$-terminal kinase (JNK) ${ }^{18}$ Lipopolysaccharide (LPS) was shown to activate a TRAF6/ASK1/p38 pathway through ROS production, indicating that this pathway has a key role in TLR4-mediated innate immunity. ${ }^{19}$ However, TLR4-mediated downstream signaling controlling apoptosis during IRI still remains not well known.

Members of the NAD(P)H oxidase (NOX) family producing ROS, which are homologues of the $\mathrm{gp}^{\text {91phox }}$ catalytic subunit of phagocytic $\mathrm{NAD}(\mathrm{P}) \mathrm{H}$ oxidase (also designated $\mathrm{NOX} 2$ ), have been shown to be involved in cellular functions related to innate immunity, signal transduction, proliferation, and/or apoptosis. ${ }^{20,21}$ NOX1, all the components of the phagocytic NOX2, and NOX4 are expressed in the kidney, NOX4 being the most abundant isoform expressed in this tissue, mainly in RTECs. ${ }^{22,23}$ NOX 4 was shown to be required for LPS-induced $\mathrm{H}_{2} \mathrm{O}_{2}$ generation in TLR4-expressing HEK 293T cells, and to interact directly with TLR4 in regulating NF- $\kappa \mathrm{B}$ activation. ${ }^{24}$ NOX4 induction has been reported in a variety of cell types subjected to ER stress, hypoxia, or ischemia, suggesting that this non-phagocytic NOX might have a role in inducing cell damage during IRI. ${ }^{20}$ In this study, we investigated the roles of gp96 and NOX4 in controlling TLR4-mediated apoptotic signaling in a murine experimental model of renal IRI, and in primary cultures of wild-type, T/r4 ${ }^{-1-}$, and $M y D 88^{-/-}$RTECs subjected to hypoxia. gp96, which interacts with TLR4 in RTECs, appears to be essential for the induction of the TLR4mediated ASK1/JNK apoptosis pathway. We also identified a NOX4 spliced isoform predominantly expressed in RTECs, which interacts with TLR4 and behaves as a key regulator of the TLR4-mediated apoptosis in posthypoxic RTECs.

\section{Results}

TLR4 mediates the activation of the TRAF2/ASK1/JNK pathway. Renal IRI caused by bilateral clamping of the renal pedicles from wild-type mice induces marked renal dysfunction and tubule cell damages, which peaked during the first 2 days after the reperfusion of the kidneys. ${ }^{9,13}$ The fact that renal dysfunction and tubule cells apoptosis were significantly less marked in T/r4 ${ }^{-/}$mice than in wild-type kidneys $^{9,13}$ led us to analyze the activation of MAPK pathways in postischemic, reperfused wild-type and TIr4 ${ }^{-/}$ kidneys. IRI increased the expression level of TRAF2, and phosphorylated ( $p-)$ ASK1 and p-JNK, and to a lesser extent p-p38, in day-1 and day-2 postischemic wild-type kidneys, but not in postischemic T/r4 ${ }^{-/}$kidneys (Figure 1a). The proapoptotic Bcl-2 homologue BAX, but not the anti-apoptotic $\mathrm{Bcl}-2,{ }^{25}$ also significantly increased in wild-type, but not in $\mathrm{Tl}_{\mathrm{r}}{ }^{-1-}$ postischemic kidneys (Figure 1a). These findings indicate that TLR4 mediates predominant activation of p-ASK1 and p-JNK, two key signal events responsible for apoptosis during IRI, ${ }^{13}$ in postischemic kidneys.

TLR4 and MyD88 mediate ROS production and apoptosis in posthypoxic renal tubule cells. Experiments were carried out on primary cultures of RTECs isolated from the kidneys of wild-type, $\mathrm{Tlr}^{-1-}$, and $M y D 88^{-1-}$ mice to analyze the pathways controlling ROS production and apoptosis in RTECs subjected to hypoxia by incubating the cells with mineral oil. ${ }^{7,13}$ The expression levels of TRAF2 over $\beta$-actin, p-ASK1, p-p38, and p-JNK, and BAX, but not of Bcl-2, were also greater in posthypoxic than in untreated wild-type RTECs (Figure 1b). In contrast, the amounts of p-ASK1, p-p38, p-JNK, and BAX remained barely detectable in posthypoxic $M y D 88^{-/-}$and remained
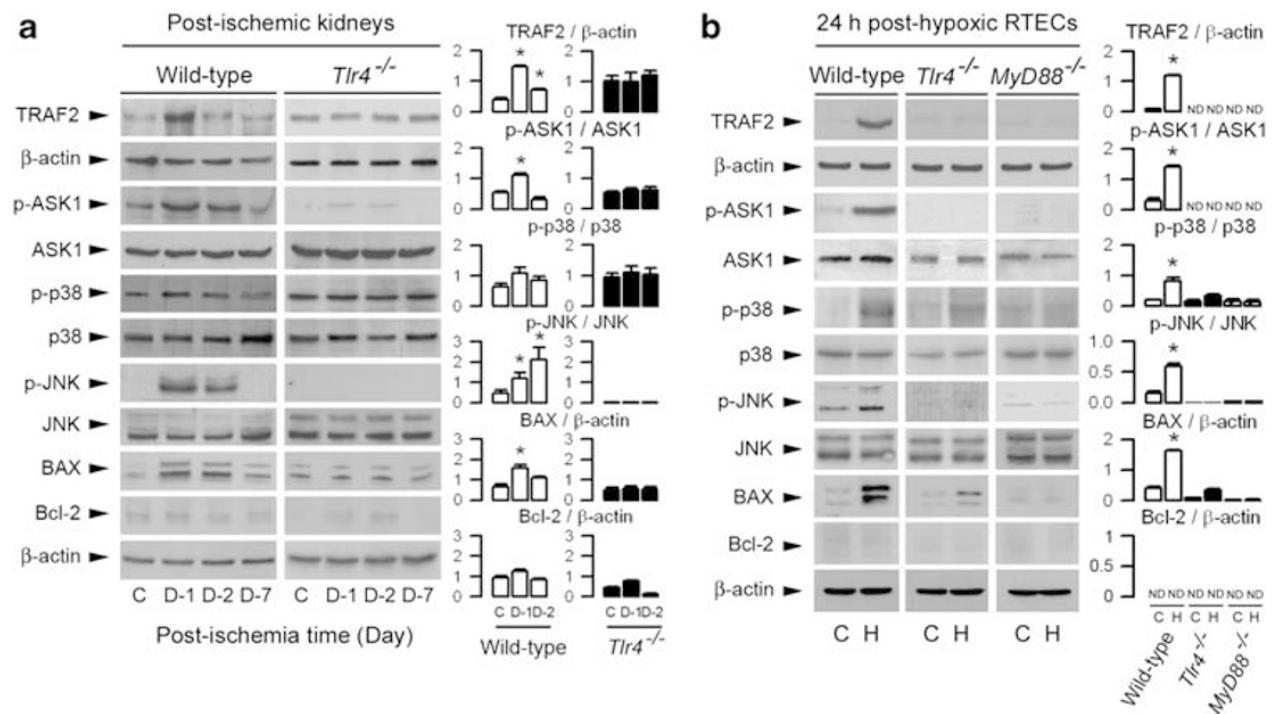

Figure 1 TLR4-dependent signaling in ischemic-reperfused kidneys and in posthypoxic renal tubule cells. Immunoblot analysis of TRAF2 and $\beta$-actin, phospho (p-) and total ASK1, p38, JNK, BAX and Bcl-2, and $\beta$-actin labeled bands in control (C), and day (D)-1, D-2, and D-7 postischemic wild-type and Tlr4 ${ }^{-1-}$ kidneys (a), and in nonhypoxic (C) and day-1 posthypoxic (H) wild-type, TIr $4^{-1-}$, and MyD88 ${ }^{-1}$ RTECs $(\mathbf{b})$. Bars are the ratio values of densitometric analyses of TRAF2, BAX, and Bcl-2 over $\beta$-actin, and p- over total ASK1, p38, and JNK labeled bands. Values are means \pm S.E.M. from three to four independent experiments. ${ }^{*} P<0.05$ versus control (C) values 
unchanged or only moderately increased in posthypoxic T/r4 ${ }^{-1-}$ RTECs (Figure 1b). As previously reported, ${ }^{9}$ the number of active caspase-3-positive apoptotic cells was significantly lower in the $24 \mathrm{~h}$ posthypoxic $\mathrm{T} / \mathrm{r} 4^{-1-}$ and $M y D 88^{-1-}$ RTECs than in wild-type RTECs (Supplementary Figure S1a and b). Imaging assays using $2^{\prime}, 7^{\prime}$ dichlorofluorescein diacetate (DCFH-DA) to measure ROS produced by intact cells ${ }^{26}$ revealed that hypoxia stimulated ROS production in wild-type RTECs, which was inhibited by adding $2 \mu \mathrm{M}$ diphenyleneiodonium chloride (DPI), a potent flavoprotein inhibitor (Supplementary Figure S1c). In contrast, the DPI-inhibitable production of ROS remained significantly lower in posthypoxic T/r4 ${ }^{-1-}$ and $M y D 88^{-1-}$ RTECs than in wild-type counterparts (Supplementary Figure S1C).

\section{Interaction of TLR4 with gp96 is required for apoptosis to be induced in posthypoxic renal tubule cells. Acute} ischemia induces misfolding of secretory proteins in the ER and increases the expression of ER chaperones triggered by ER stress signaling pathways. gp96, one of the most abundant Hsps residing in the ER that is induced by the accumulation of misfolded proteins caused by cellular stress, has an essential role in the maturation and proper folding of membranous and intracellular TLRs. ${ }^{12,27}$ The amount of gp96 significantly increased in both wild-type and T/r4 ${ }^{-1-}$ RTECs subjected to hypoxia (Figure 2a). Coimmunoprecipitation (co-IP) studies revealed that TLR4 interacts with gp96 in lysates from nonhypoxic wild-type RTECs (Figure 2b) or established mouse renal proximal tubule PKSV-PR cells ${ }^{28}$ (not shown). Co-IP experiments also showed that hypoxia did not disrupt the interaction between gp96 and TLR4 (Figure 2c). We next analyzed the consequences of gp96 extinction on hypoxia-induced proapoptotic signaling. Knockdown gp96 mRNA expression by silencing RNA (siRNA) (Figure 2d) had no significant effect on p-ASK1 or p-JNK in nonhypoxic wild-type RTECs (not shown), but in contrast, it did prevent the increase in TRAF2, p-ASK1, and p-JNK in posthypoxic wild-type RTECs (Figure 2e). ROS production and induction of apoptosis were also no longer observed in gp96 siRNA-transfected RTECs subjected to hypoxia when compared to nontransfected posthypoxic RTECs or cells transfected with the control siRNA (Figure $2 f$ and $g$ ). Transfecting cells with increasing concentrations of gp96 siRNA also showed that about $70 \%$ inhibition of gp96 protein content was associated with an almost complete inhibition ( $>90 \%$ ) of the hypoxiainduced ROS production and about $80 \%$ inhibition of apoptosis in wild-type RTECs (Supplementary Figure S2a and b). Silencing gp96 was shown to reactivate the expression of ERK1/2, but not of ASK1 and JNK, in posthypoxic T/r2 ${ }^{-1-}$ RTECs. ${ }^{13}$ In contrast to the effect of PP5 silencing mRNA expression on the reinduction of apoptosis in posthypoxic TIr2 ${ }^{-1}$ RTECs, which occurs through reactivation of the $p$-ASK1/p-JNK pro-apoptotic pathway, ${ }^{13}$ silencing gp96 did neither induce reactivation of ROS production nor induction of apoptosis in gp96 siRNAtransfected posthypoxic TIr $4^{-1-}$ RTECs or TIr2 ${ }^{-1-}$ RTECs (Figure $2 \mathrm{~h}$ and $\mathrm{i}$ ). Because the expression levels of TRAF2 had increased in postischemic wild-type kidneys, its participation in the activation of apoptosis was analyzed. Co-IP experiments revealed that TRAF2, which is not associated with ASK1 in nonhypoxic RTECs, coimmunoprecipitated with ASK1 in posthypoxic wild-type RTECs (Figure 3a). Furthermore, silencing Traf2 mRNA expression almost fully impaired the activation of NOX4, pASK1 and p-JNK, ROS production, and apoptosis in posthypoxic wild-type RTECs (Figure $3 b-d$ ).

IRI induces NOX4 overexpression in renal tubule cells. Because NOX enzymes are involved in the regulation of a wide range of physiological functions, including cell death, ${ }^{20}$ experiments were carried out to investigate the consequences of $|R|$ on the renal expression of NOX1 and NOX4, which are both predominantly expressed in the kidney. ${ }^{20,22,23}$ Western blot analyses revealed the same $\sim 65-\mathrm{kDa}$ NOX1 protein band of the expected size ${ }^{29}$ in homogenates of mouse kidney and colon (Figure 4a). Western blot analysis using an antibody raised against the $\mathrm{C}$-terminal region of mouse NOX4 revealed a major $\sim 65-\mathrm{kDa}$ band similar to NOX1 and a minor $28-\mathrm{kDa}$ band in the colon homogenate (Figure $4 \mathrm{~b}$ ). In sharp contrast, the $28-\mathrm{kDa}$ NOX4 was the major protein band detected in the kidney homogenate, whereas the $65-\mathrm{kDa}$ NOX4 remained barely detectable (Figure $4 b$ ), suggesting that the 28-kDa NOX4 is the main NOX4 isoform expressed in the mouse kidney. Four human NOX4 splice variants have been identified, including a NOX4 D variant lacking exons 3-11 with a predicted molecular weight of $28 \mathrm{kDa} .^{30} \mathrm{We}$ identified the full-length Nox4 (encoding for the 65-kDa NOX4 protein) and a similar Nox4 variant in the mouse kidney (Supplementary Figure $\mathrm{S} 3 \mathrm{a}$ ) and in the murine

\footnotetext{
Figure 2 gp96 interacts with TLR4 to produce the proper induction of ROS production and apoptosis in posthypoxic renal tubule cells. (a) Immunoblot analyses of gp96 and the corresponding $\beta$-actin in nonhypoxic (C) and posthypoxic (H) wild-type (WT) and T/r $4^{-1-}$ RTECs. (b and c) Lysates from nonhypoxic (C) or $24 \mathrm{~h}$ posthypoxic (H) WT RTECs were subjected to IP using an antibody against TLR4 (b) or gp96 (c). The IP material was then subjected to western blot analysis, and proteins were detected with antigp96 or anti-TLR4 antibodies (b and c). As controls, western blotting of non-immunoprecipitated cell lysates (none) revealed the presence of equal amounts of TLR4 or gp96 in the cell lysates subjected to immunoprecipitation. (d) Immunoblot analyses of gp96 and the corresponding $\beta$-actin in day-1 posthypoxic (hypoxia) WT RTECs that had or had not been transfected with a gp96 siRNA or negative control siRNA. (e) Immunoblot (left panel) and densitometric analyses (right panel) (representative of three separate experiments) of TRAF2 and the corresponding $\beta$-actin, and phosphorylated (p-) over total ASK1 and JNK in day-1 posthypoxic (hypoxia) WT RTECs that had or had not been transfected with the gp96 siRNA or negative control siRNA. ( $\mathbf{f}$ and $\mathbf{g})$ DCF fluorescence recordings of ROS production (f), and percentage of apoptotic cells ( $\mathbf{g}$ ) in control and day-1 posthypoxic (hypoxia) wild-type RTECs that had or had not been transfected with the gp96 siRNA or negative control siRNA. (h and i) DCF fluorescence recordings of ROS production (h), and percentage of apoptotic cells (i) in $24 \mathrm{~h}$ posthypoxic (hypoxia) wild-type, $T / \mathrm{r} 4^{-/-}$, and TIr2 ${ }^{-1-}$ RTECs transfected or not with the gp96 siRNA. Values are means \pm S.E.M. from $(n)$ individual cell recordings or cell counts performed on 2-3 separate cell cultures from two ( $h$ and $\mathbf{i}$ ) to three (f and $\mathbf{g}$ ) different kidneys for each condition tested. ${ }^{*} P<0.05$ between groups
} 


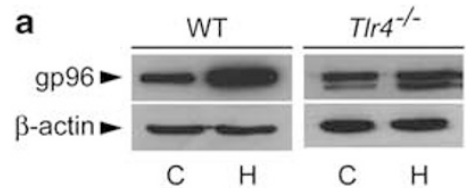

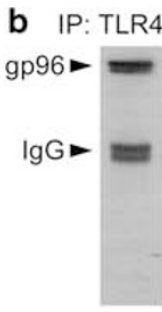

C

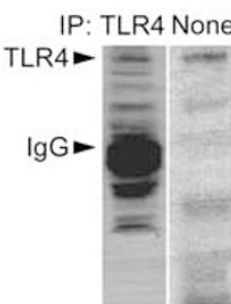

C C

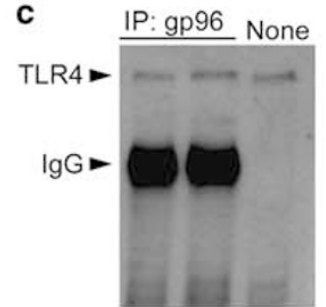

C $\mathrm{H} \quad \mathrm{C}$

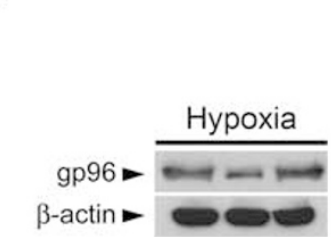

gp96 siRNA - + -
Hypoxia

e

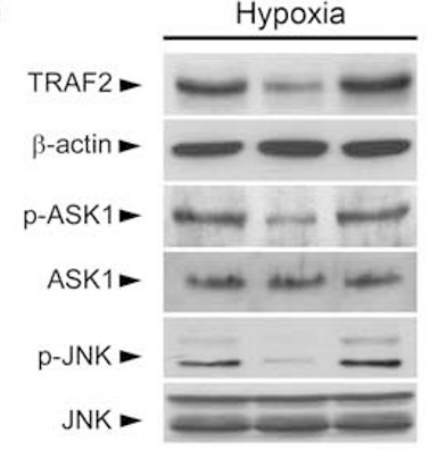

IP: gp96 None

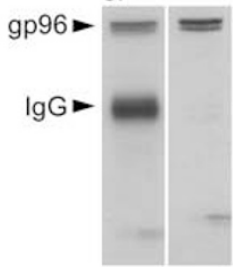

C C

Control siRNA - -+

gp96 siRNA

$-$

$+$
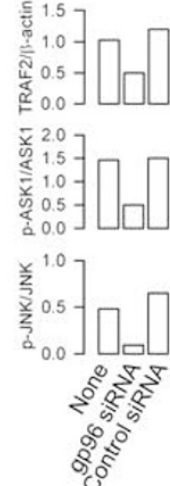

f

$\square$ Control $\mathbf{a y p o x i a} \quad \mathbf{g}$ Wild-type RTECs
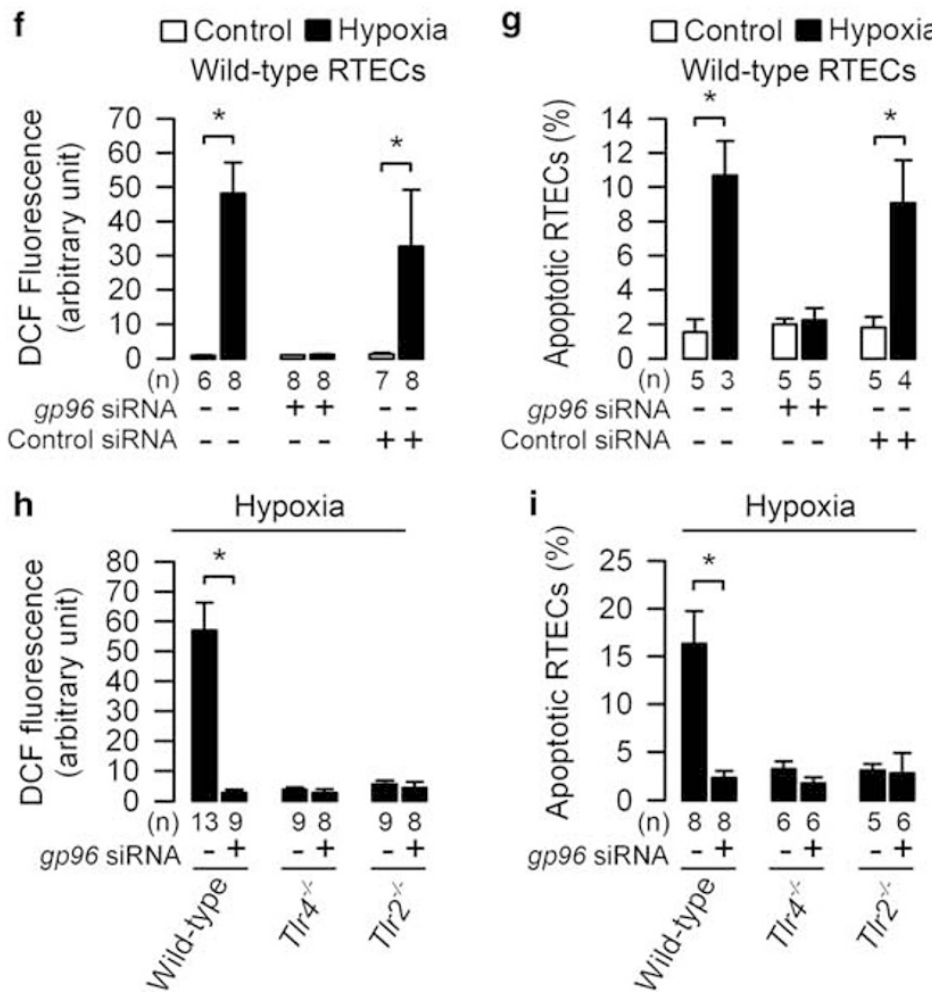
proximal tubule PKSV-PR cells using different sets of primers within mouse NOX4 exons 1 and 14 (Supplementary Figure S3b). The fact that TLR4 was shown to interact with human NOX4 in TLR4-transfected HEK 293T cells ${ }^{24}$ led us to test whether endogenous mouse TLR4 interacted with the 28-kDa NOX4, and whether the 28-kDa NOX4 interacted with gp96. TLR4 co-immunoprecipitated with the $28-\mathrm{kDa}$ NOX4 and gp96 in nonhypoxic and posthypoxic wild-type RTEC lysates (Figure $4 \mathrm{c}$ and $\mathrm{d}$ ). The amount of gp96 and NOX4 bound to TLR4 was greater in posthypoxic lysates than in nonhypoxic lysates, whereas the amount of TLR4 bound to immunoprecipitated gp96 remained unchanged or only moderately increased in posthypoxic RTECs (Figures $2 \mathrm{c}$ and $4 \mathrm{e}$, left panel). These findings thus suggest that hypoxia stimulates the binding of gp96 and NOX4 to TLR4. gp96 co-immunoprecipitated with NOX4 in nonhypoxic and posthypoxic wild-type RTECs (Figure 4e, left panel), but, in sharp contrast, gp96 no longer co-immunoprecipitated with NOX4 in $\mathrm{TIr}^{-1}{ }^{-}$RTECs (Figure $4 \mathrm{e}$, right panel), indicating that the $28-\mathrm{kDa}$ NOX4 isoform does not directly interact with gp96. Co-IP also confirmed that TLR4 co-immunoprecipitated with both 28-kDa NOX4 and gp96 in nonhypoxic and posthypoxic wild-type RTECs (Figure 4f), suggesting that they form a trimeric complex (Figure $4 \mathrm{~g}$ ).

We next analyzed the expression of NOX4 compared to that of NOX1 in postischemic wild-type kidneys. At 1 and 2 days after IRI, the amount of the 28-kDa NOX4 isoform had increased whereas that of NOX1 had decreased (Figure $4 \mathrm{~h}$ ). The upregulation of the 28-kDa NOX4 appears to be closely related to TLR4-dependent renal injury, because the increase in NOX4 was not detected in postischemic Tlr4 ${ }^{-/-}$kidneys (Figure 4h). Immunohistochemical analyses revealed cellular punctuate immunostaining of NOX1 evenly distributed in all tubule cell sections, and NOX4 immunostaining preferentially restricted to some tubule cell sections (Figure $4 \mathrm{i}$, upper panels). At $48 \mathrm{~h}$ after ischemia, NOX1 was barely detectable in tubule cell sections, whereas intense NOX4 labeling was detected in all tubule cell sections from the medulla (Figure $4 \mathrm{i}$, lower panels). I/R induces potent inflammatory response, and the influx of neutrophils and macrophages into injured kidneys. $^{7,9}$ NOX2, which is expressed in neutrophils, ${ }^{20,21}$ but not in RTECs, greatly increased after IRI in wild-type kidneys, but not in postischemic T/r4 ${ }^{-/}$kidneys (Supplementary Figure S4a). However, NOX2 did not seem to have any significant role in ischemia-induced apoptosis, because the number of terminal deoxyribonucleotide transferase (TdT)-mediated dUTP nick end labeling (TUNEL)-positive cells did not significantly differ in postischemic Nox2 ${ }^{-1-}$ and wild-type kidneys (Supplementary Figure S4b). These findings indicate that the renal $28-\mathrm{kDa}$ NOX4 spliced isoform is upregulated after IRI, suggesting that this NOX4 isoform is involved in the induction of tubule cell apoptosis in injured kidneys after IRI. a

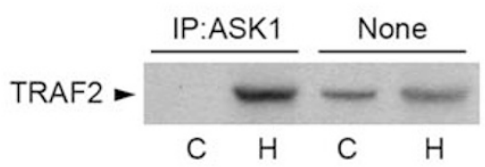

b

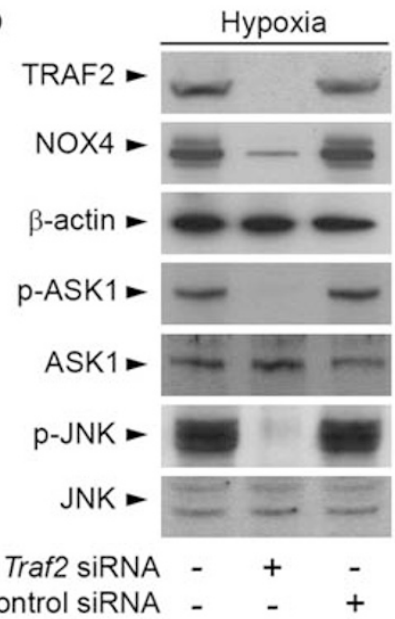
2 c

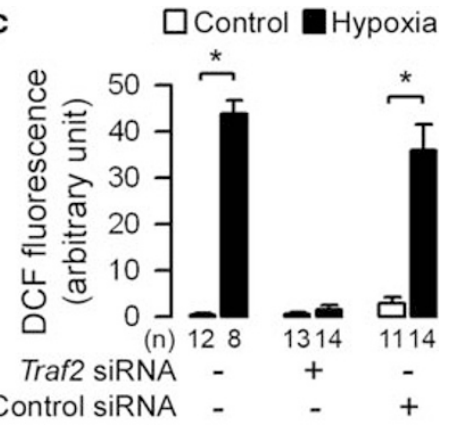

d

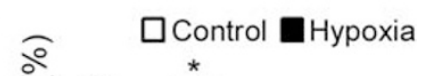

-ASK1 / ASK1
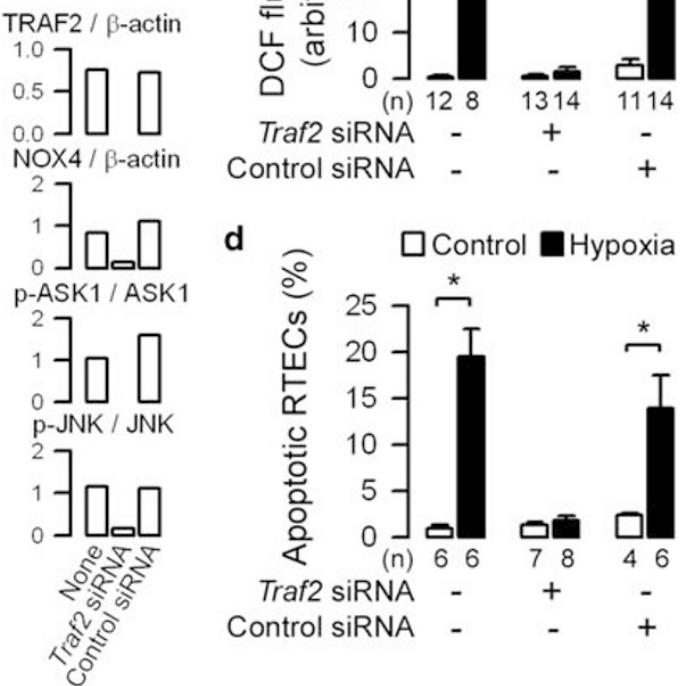

Figure 3 TRAF2 controls phosphorylated ASK1 and JNK in posthypoxic renal tubule cells. (a) Lysates from nonhypoxic (C) or $24 \mathrm{~h}$ posthypoxic (H) wild-type RTECs were subjected to IP using an antibody against ASK1. The IP material was then subjected to western blot analysis, and proteins were detected with an anti-TRAF2 antibody. (b) Immunoblot (left panel) and densitometric analyses (right panel) (representative of two separate experiments) of TRAF2 and NOX4, and corresponding $\beta$-actin, and phosphorylated ( $p$-) over total ASK1 and JNK in day-1 posthypoxic (hypoxia) wild-type RTECs transfected or not with a specific Traf2 siRNA or negative control siRNA. (c and d) DCF fluorescence recordings of ROS production (c), and percentage of apoptotic cells (d) in control and day-1 posthypoxic (hypoxia) wild-type RTECs that had or had not been transfected with a specific Traf2 siRNA or negative control siRNA. Values are means \pm S.E.M. from $(n)$ individual cell recordings or cell counts performed on two separate cell cultures from two different kidneys for each condition. ${ }^{*} P<0.05$ between groups 

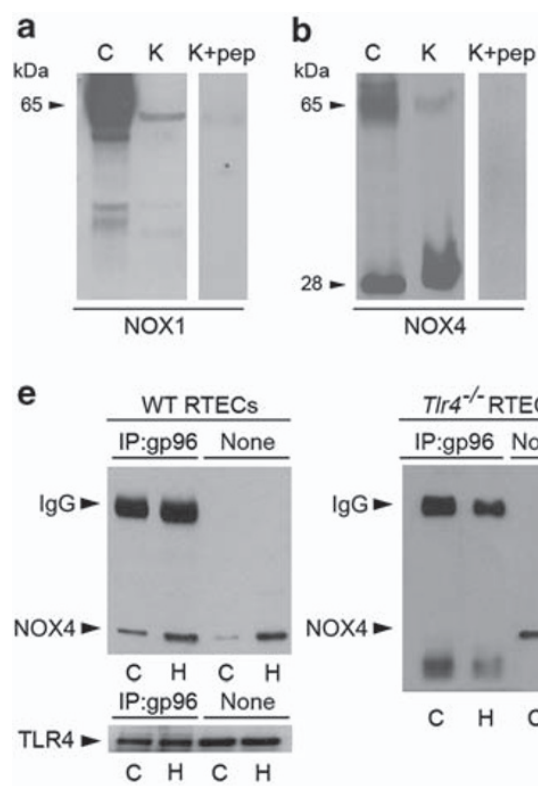

h

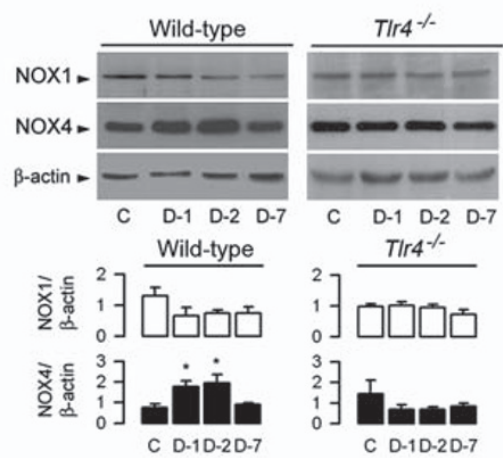

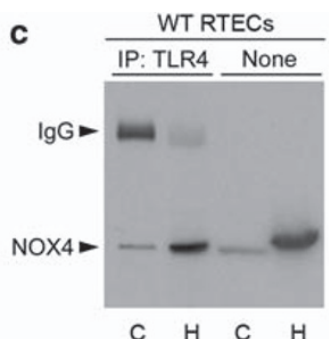
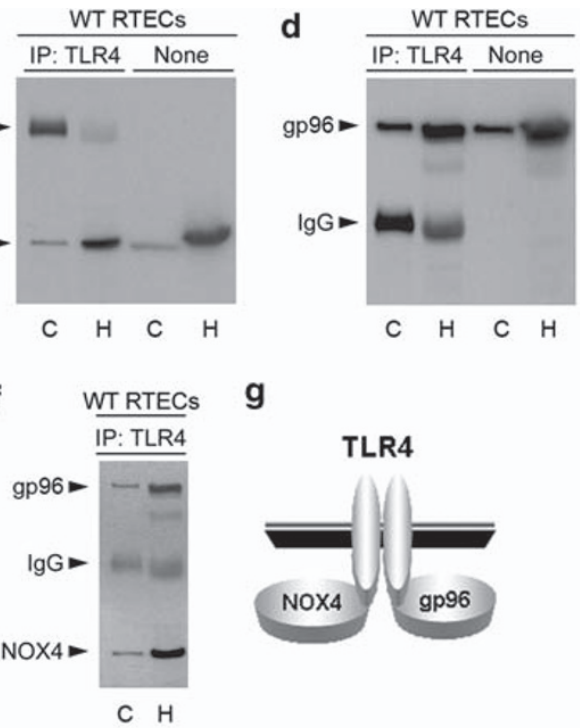

g

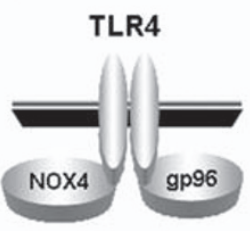

i
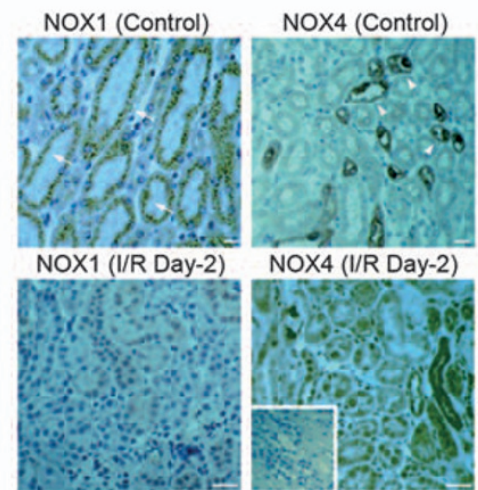

Figure 4 Renal ischemia/reperfusion injury induces selective overexpression of NOX4 interacting with TLR4 in wild-type mice kidneys. (a and $\mathbf{b})$ Immunoblot analysis of NOX1 (a) and NOX4 (b) in colon (C) and kidney (K) homogenates that had or had not been incubated with an excess of the peptide used for rabbit immunization (K+ pep). (c-f) Lysates from nonhypoxic (C) or $24 \mathrm{~h}$ posthypoxic (H) wild-type (WT) RTECs or TIr4 ${ }^{-/}$RTECs were subjected to IP using an antibody against TLR4 (c, d, f) or gp96 (e). The IP material was then subjected to western blot analysis, and proteins were detected either with anti-NOX4 (c, e, f), anti-TLR4 (e), and/or anti-gp96 (d and f) antibodies. As controls, western blotting analyses were performed on non-immunoprecipitated cell lysates (none). (g) Schematic representation of the TLR4/gp96/NOX4 complex. (h) Immunoblot analysis of NOX1, NOX4, and $\beta$-actin in control (C) and day (D)-1, D-2, and D-7 postischemic wild-type and TIr $4^{-1-}$ kidney homogenates. Bars are the ratio values (means \pm S.E.M. from three to five separate experiments) of densitometric analyses of NOX1 or NOX4 over $\beta$-actin labeled bands. ${ }^{*} P<0.05$ versus control (C) values. (i) Patterns of NOX1 (arrows) and NOX4 (arrowheads) immunostaining in nonischemic (control, upper panels) and day-2 postischemic (l/R Day-2, lower panels) WT kidneys. As a control, no positive NOX4 immunostaining was detected in the day-2 postischemic kidney section incubated with an excess of the peptide used for immunization (i, inset). $\mathrm{Bar}=10 \mu \mathrm{m}$

Interaction between TLR4 and 28-kDa NOX4 is required for ROS production and for apoptosis in posthypoxic renal tubule cells. The interaction between TLR4 and NOX4 was shown to be essential for the LPS-induced production of ROS to occur. ${ }^{24}$ The question arises as to whether the 28-kDa NOX4 isoform identified in the mouse kidney might have a role in TLR4-mediated ROS production and apoptosis during IRI. NOX4 is considered to be a constitutive ROS-generating NOX. ${ }^{20,31}$ However, the ability of the $28-\mathrm{kDa}$ NOX4 variant to generate ROS remains elusive. Experiments were carried out on posthypoxic wildtype RTECs transfected or not with various Nox4 siRNAs or a control siRNA to further assess the role of the $28-\mathrm{kDa}$ NOX4 isoform in TLR4-mediated apoptosis. Silencing both the full-length $65-\mathrm{kDa}$ and $28-\mathrm{kDa}$ Nox4 mRNAs using two siRNAs that target the sequences of exons 1 and 3 (designated as Nox4 ${ }_{65-28}$ siRNA) inhibited by more than $80 \%$ the increase in both 28- and 65-kDa NOX4 mRNA and protein expressions (Figure $5 a$ and $b$, left panels), ROS production, and apoptosis caused by hypoxia (Figure $5 \mathrm{c}$ ). Similarly, silencing the 28-kDa Nox4 isoform using an siRNA directed against a target sequence overlapping exons 2 and 12 (designated as $\mathrm{Nox}_{28}$ siRNA) (Figure 5a, right panel), which selectively blunted the expression of the 28-kDa NOX4 protein by about $60 \%$ (Figure $5 \mathrm{a}$ and $\mathrm{b}$, right panel), led to a almost complete inhibition of ROS production and about $80 \%$ inhibition of apoptosis in posthypoxic RTECs (Figure 5c). Consistent with these findings, transfecting cells with various 
a

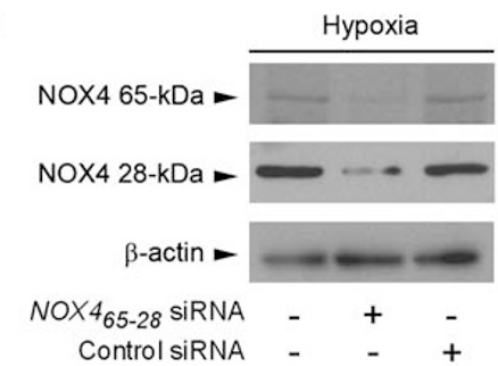

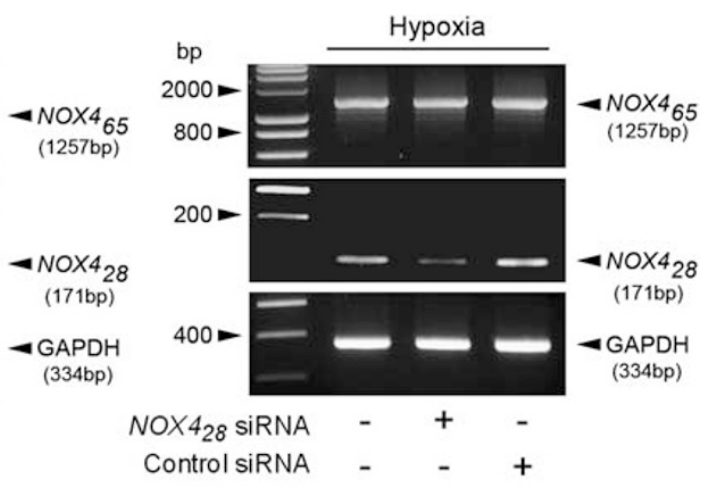

Hypoxia

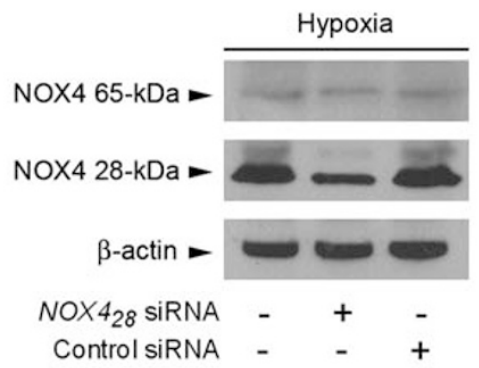

c

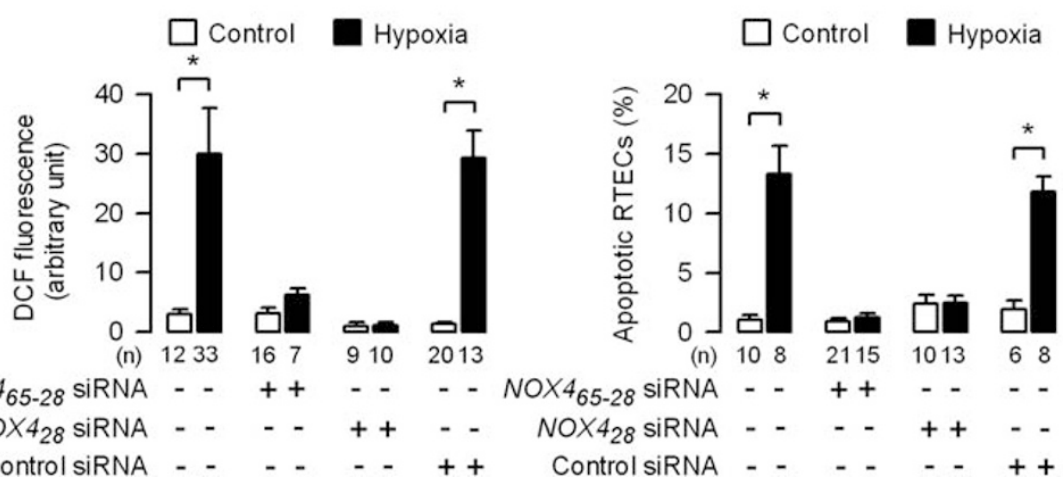

Figure 5 The 28-kDa NOX4 controls apoptosis in posthypoxic renal tubule epithelial cells. (a and $\mathbf{b}) \mathrm{RT}$-PCR analysis of the Nox4 mRNA isoforms and corresponding GAPDH mRNA expression (a), and immunoblot analysis of the $65-\mathrm{kDa}$ (b) upper panels) and 28-kDa NOX4 protein (b, middle panels) isoforms and corresponding $\beta$-actin (b, lower panels) in primary cultures of $24 \mathrm{~h}$ posthypoxic wild-type RTECs that had or had not been transfected with specific Nox4 siRNAs, which target sequences encoding either both the full-length $65-\mathrm{kDa}$ and the $28-\mathrm{kDa}$ isoform (Nox465-28) (left panels) or only the $28-\mathrm{kDa}$ NOX4 isoform (Nox4 $\left.{ }_{28}\right)$ (right panels). As controls, posthypoxic RTECs were also transfected with a negative control siRNA (data are representative of three separate experiments). (c) DCF fluorescence recordings of ROS production (left panel) and percentage of apoptotic cells (right panel) in nonhypoxic (control) and day-1 posthypoxic (hypoxia) wild-type RTECs transfected or not with the two Nox4 siRNAs, or with negative control siRNA. Values are means \pm S.E.M. from $(n)$ individual cell recordings or cell counts performed on two separate cell cultures for each condition tested. ${ }^{*} P<0.05$ between groups

concentrations of the $N O X 4_{28}$ siRNA led to a concentrationdependent inhibition in both ROS production and apoptosis caused by hypoxia (see Supplementary Figure S2). These data further suggest that the $28-\mathrm{kDa}$ NOX4 isoform is required in the induction of TLR4-mediated apoptosis in posthypoxic RTECs.

NOX4 controls JNK-mediated renal tubule cell apoptosis induced by hypoxia. We next investigated the role of the 28-kDa NOX4 in inducing the TRAF2/ASK1/JNK proapoptotic pathway in posthypoxic, wild-type RTECs. As in postischemic kidneys, the amount of the 28-kDa NOX4, but not that of NOX1, increased significantly in the $24 \mathrm{~h}$ posthypoxic, wild-type RTECs, but not in posthypoxic
TIr4 ${ }^{-1-}$ or MyD88 ${ }^{-1-}$ RTECs (Figure 6a). The Nox4 28 siRNA did not impair the expression levels of TRAF2 in posthypoxic, wild-type RTECs, but did reduce the hypoxiainduced p-ASK1 and p-JNK activation in posthypoxic RTECs (Figure 6b). In contrast, the stimulation of NOX4, p-ASK1, p-JNK, ROS production, and apoptosis was not altered in posthypoxic Nox1 ${ }^{-/-}$RTECs (Supplementary Figure S5). Wild-type RTECs were then incubated with the JNK inhibitor SP600125 to test whether JNK could reciprocally regulate NOX4 and inhibit apoptosis. Pre-incubating cells with SP600125 $(2 \mu \mathrm{M})$, which did not affect the activation of the other MAPK kinases (Supplementary Figure S6a), reduced the activation of the c-Jun, the substrate of JNK, and NOX4 (Figure 6c). Moreover, SP600125 significantly inhibited ROS 

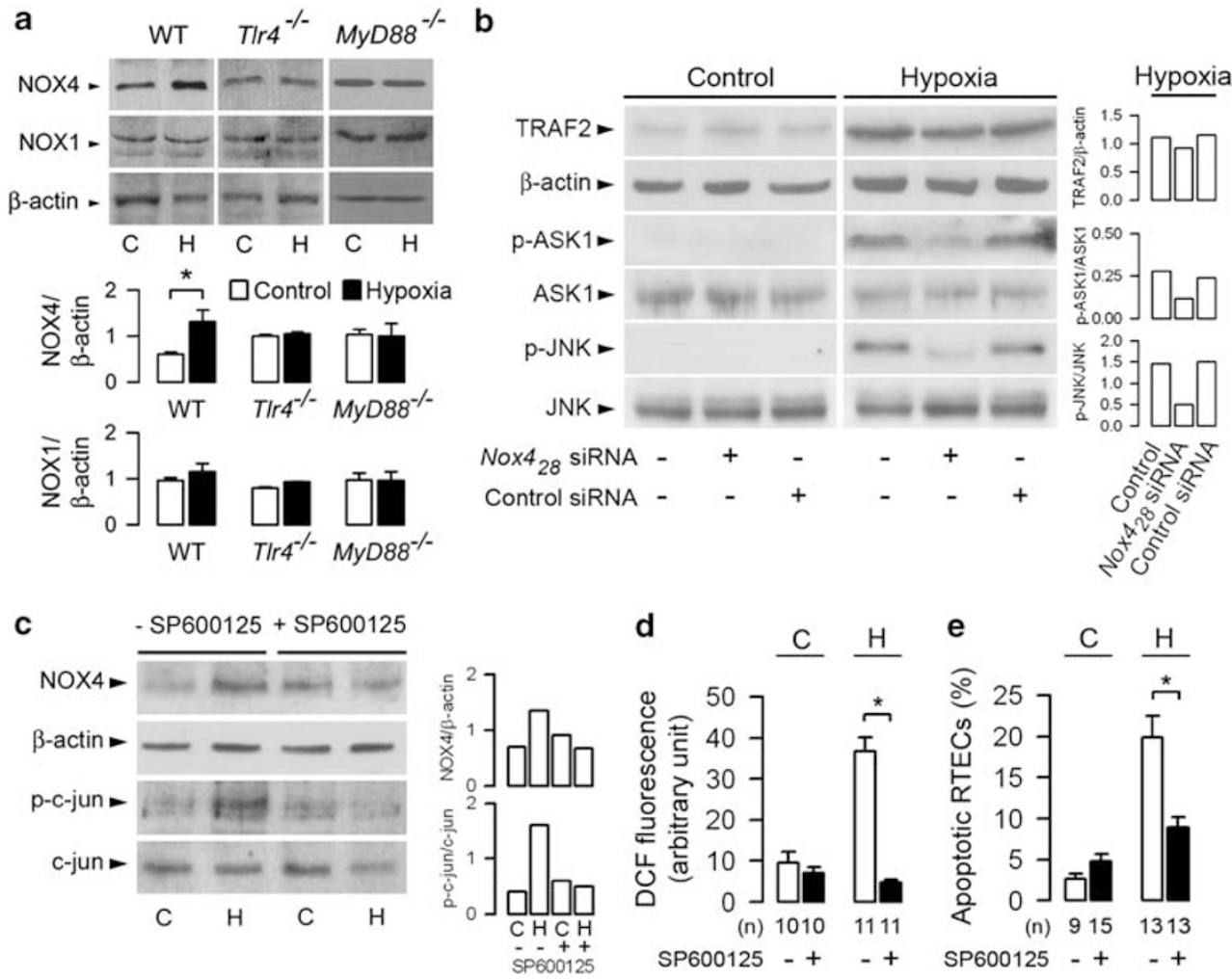

Figure 6 Reciprocal control of 28-kDa NOX4 and JNK on ROS production and apoptosis in posthypoxic renal tubule cells. (a) Immunoblot analysis (upper panel) and densitometric values (lower panel) (means \pm S.E.M. from three to five separate experiments) of NOX4, NOX1, and corresponding $\beta$-actin in control (C) and day-1 posthypoxic

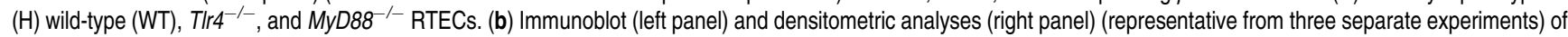
TRAF2 and corresponding $\beta$-actin, p-, and total ASK1 and JNK in control and day-1 posthypoxic (hypoxia) wild-type RTECs that had or had not been transfected with the NOX4 $4_{28}$ siRNA or negative control siRNA. (c-e) Immunoblot and densitometric analyses (representative from two separate experiments) of the 28-kDa NOX4 and corresponding $\beta$-actin, $\mathrm{p}$-, and total c-jun (c), DCF fluorescence recordings (d), and percentage of apoptotic cells (e) in control (C) and day-1 posthypoxic (H) wild-type RTECs incubated with $(+)$ or without $(-) 2 \mu \mathrm{M} \mathrm{SP} 600125 \mathrm{JNK}$ inhibitor that was added $30 \mathrm{~min}$ before adding mineral oil and during the $24 \mathrm{~h}$ after hypoxia induction. Values are means \pm S.E.M. from $(n)$ individual cell recordings or cell counts on 3-5 cell cultures from two to three different kidneys in each group tested. ${ }^{*} P<0.05$ between groups

production and the percentage of apoptotic cells, respectively, caused by hypoxia (Figure $6 \mathrm{~d}$ and $\mathrm{e}$ ). Altogether, these findings indicate that NOX4 controls the activation of the JNK/ASK1 pro-apoptotic pathway in posthypoxic RTECs.

Silencing NOX4 impairs the activation of p38 in posthypoxic renal tubule cells. Because hypoxia induced the activation of p-p38, together with that of p-ASK1 and p-JNK, in posthypoxic RTECs, the question arises as to whether NOX4 also controls the activation of p38. Silencing $N o x 4_{28}$ siRNA expression, in addition to its inhibitory action on $\mathrm{p}$-ASK1 and p-JNK, fully inhibited the activation of p-p38 in posthypoxic wild-type RTECs (Figure 7a). We then tested the consequence of p38 activity inhibition on ROS production and apoptosis. RTECs were incubated with $10 \mu \mathrm{M}$ of the selective p38 inhibitor SB203580, which prevented the activation of the p38 substrate MAPKAPK2 (Figure 7b) without affecting the activation of the other MAPK kinases (Supplementary Figure S6b). SB203580 significantly reduced the hypoxiainduced ROS production (Figure $7 \mathrm{c}$ ), but had only a borderline significant inhibitory action on apoptosis in posthypoxic RTECs (Figure 7d). Because the selective inhibition of p38 did not impair the activation of p-JNK, we tested whether the inhibition of both p38 and JNK activity could fully impair cell apoptosis induced by hypoxia. Preincubation of wild-type RTECs with $2 \mu \mathrm{M}$ SP600125 in addition to $10 \mu \mathrm{M}$ SB203580 was more efficient than SP600125 alone or SB203580 alone to inhibit apoptosis in posthypoxic wild-type RTECs (Figures $6 \mathrm{e}$ and 7d). Collectively, these findings indicated that induction of apoptosis in posthypoxic RTECs results from the combined activation of ASK1 and both JNK and p38 MAPKs.

\section{Discussion}

$I R I$ induces the activation of the innate immune response that leads to a rapid inflammatory response and apoptosis. Previous studies indicate that both TLR4 and TLR2 have central roles in the pathogenesis of renal IRI ${ }^{7-9}$ and that TLR4/ MyD88 signaling contributes to the induction of RTEC apoptosis. ${ }^{9}$ In this study, we show that TLR4 complexed with gp96 mediates the pro-apoptotic TRAF2/ASK1/JNK pathway in posthypoxic RTECs. We also found that the non-phagocytic 28-kDa NOX4 isoform, which interacts with TLR4, has a 
a

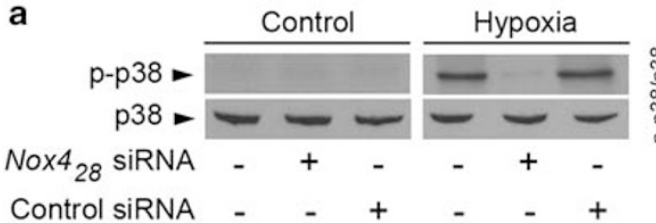

b $\frac{\text { Wild-type RTECs }}{- \text { SB203580 + SB203580 }}$
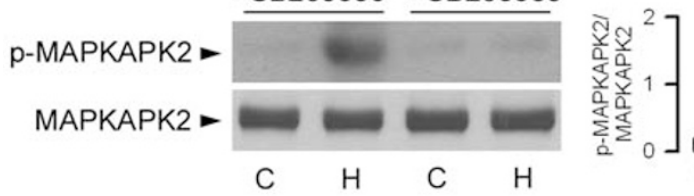

C

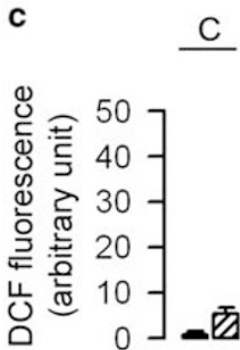

(n) 912 SB203580 _ + ++
Hypoxia

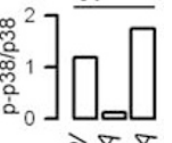

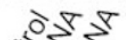
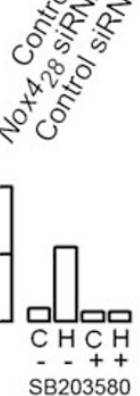

Figure 7 Effects of silencing the NOX4 28-kDa isoform on p38 activation and consequence of p38 inhibition on ROS production and apoptosis in posthypoxic renal tubule cells. (a) Immunoblot (left panel) and densitometric analyses (right panel) (representative from two separate experiments) of p- and total p38 in nonhypoxic (control) and day-1 posthypoxic (hypoxia) wild-type RTECs that had or had not been transfected with the $N O X 4_{28}$ siRNA or negative control siRNA. (b) Immunoblot and densitometric analyses (representative from two separate experiments) of $\mathrm{p}$ - and total MAPKAPK2 in control $(\mathrm{C})$ and day-1 posthypoxic $(\mathrm{H})$ wild-type RTECs incubated (+) or not (-) with $10 \mu \mathrm{M}$ SB203580 p38 inhibitor as described in Figure 6. (c) DCF fluorescence recordings in nonhypoxic control $(\mathrm{C})$ and day-1 posthypoxic $(\mathrm{H})$ wild-type RTECs incubated without $(-)$ or with $(+)$ $10 \mu \mathrm{M}$ SB203580. (d) Percentage of apoptotic cells in nonhypoxic control (C) and day-1 posthypoxic $(\mathrm{H})$ wild-type RTECs incubated without $(-)$ or with $(+) 10 \mu \mathrm{M}$ SB203580 alone or with $2 \mu \mathrm{M}$ SP600125. Values are means \pm S.E.M. from $(n)$ individual cell recordings or cell counts on 2-3 cell cultures from two different kidneys. ${ }^{\star} P<0.05$ between groups

central role in controlling TLR4-mediated ROS production and apoptosis of posthypoxic RTECs.

The accumulation of unfolded proteins in the $\mathrm{ER}$, as a result of ER stress, induces a compensatory unfolded protein response (UPR) to permit cell survival, but if this response is not controlled properly it can also trigger cell death. ${ }^{32}$ The accumulation of misfolded proteins, like that of other Hsps, stimulates the production of gp96, ${ }^{33}$ although not essential for cell viability, ${ }^{27}$ controls the proper folding, and export of most of the TLRs, including TLR4, in a pre-B cell line and macrophages. ${ }^{13,27}$ We show that TLR4 co-immunoprecipitated with both gp96 and NOX4 and that hypoxia triggered the binding of TRAF2 to ASK1. Furthermore, silencing gp96 or Traf2 mRNA expression both impaired the upregulation of $p$ ASK1 and p-JNK, ROS production, and the induction of RTEC

apoptosis caused by hypoxia. Overall, these findings strongly suggest that the TLR4-gp96 complex is required for the proper transduction of the TLR4-mediated TRAF2/ASK1/JNK pathway in posthypoxic renal cells.

There is growing evidence that ROS are important intracellular intermediates involved in regulating cytokine expression, cell proliferation, and apoptosis. ROS can be produced by the mitochondrial respiratory chain, xanthine oxidase, xanthine oxygenase, lipoxygenase, and NOX enzymes in stressed cells. ${ }^{34}$ Functional TLR4 signaling has been shown to be required for ROS production, and subsequent release of endogenous HMGB1 in hepatocytes subjected to hypoxia. ${ }^{4}$ Here, we show that the $28-\mathrm{kDa}$ NOX4 isoform, similar to the human NOX4 D variant previously identified in human lung A549 cells and lung tissue, ${ }^{30}$ represents the main NOX4 isoform expressed in mouse RTECs. The fact that ROS production is significantly reduced in transfected posthypoxic wild-type RTECs with the selected $\mathrm{Nox}_{28}$ siRNA, which targets the 28-kDa NOX4 isoform, indirectly suggests that this NOX4 isoform is involved in the ROS production induced by hypoxia. Human NOX4 has been shown to be a constitutive ROS-generating enzyme that requires the membrane-associated subunit p22 $^{\text {phox }},{ }^{35}$ but, unlike NOX1, NOX2, or NOX3, does not require the presence of organizers NOXAs and NOXOs subunits. ${ }^{20}$ NOX4 is thought to be an inducible NOX isoform regulated at the mRNA level. ${ }^{31}$ NOX4 expression has been shown to be increased by transforming growth factor- $\beta 1$ in human pulmonary smooth muscle cells and by angiotensin II in mesangial cells (reviewed by Bedard and Krause ${ }^{20}$ ). Here we show that the mouse 28-kDa NOX4 isoform is upregulated in postischemic kidneys and posthypoxic RTECs. However, the exact mechanism by which this NOX4 variant, which lacks most transmembrane domains, interferes in ROS production remains to be determined. Furthermore, it may not in fact directly control the production of ROS induced by hypoxia. LPS-induced ROS generation and NF- $\kappa$ B activation have been shown to be mediated by direct interaction between the C-terminal region of NOX4 and the cytoplasmic tail of TLR4. ${ }^{24}$ Because the endogenous 28-kDa NOX4 isoform also coimmunoprecipitated with TLR4 in RTECs, the lack of ROS production and apoptosis observed after silencing the $28-\mathrm{kDa}$ NOX4 may reflect the efficient protection of RTECs against hypoxia due to the absence of TLR4-mediated activation, and the subsequent activation of ROS produced by pathways other than that involving ROS-generating NOX4. NOX4 that is complexed with TLR4, but not with gp96, appears to be an important effector of the ASK1/JNK pathway that is activated in response to hypoxia-induced ER stress. The fact that JNK exerts reciprocal control over NOX4, ROS production, and apoptosis in posthypoxic RTECs, further confirms the role of the TLR4-mediated TRAF2/ASK1/JNK pathway in controlling apoptosis.

Matsuzawa et al. ${ }^{19}$ showed that the ROS-dependent activation of the TRAF6/ASK1/p38 pathway is required in TLR4-mediated cell activation. Here we show that p-p38 is activated in posthypoxic RTECs, and to a lesser extent in postischemic intact kidneys. We also show that silencing $N O X 4_{28}$ mRNA expression inhibited both the activation of p-ASK1, p-JNK, and p-p38 in posthypoxic RTECs, suggesting 
that NOX4 controls the ASK1 downstream effectors p-38 and JNK. In accordance with these findings, selective inhibition of the two JNK and p38 MAPKs leads to marked inhibition in cell apoptosis caused by hypoxia. In vivo administration of the antioxidant selenite was reported to impair the rapid activation of ASK1/MAKK3/p38 signaling cascade and RTECs apoptosis $3 \mathrm{~h}$ after the reperfusion of adult male rat kidneys subjected to ischemia for $45 \mathrm{~min}^{36}$ Because selenite inhibited the activation of $p-A S K 1$, it cannot be excluded that this antioxidant agent, in addition to its inhibitory action on p38, also inhibited the activation of $\mathrm{p}$-JNK during renal IRI. However, the effect of selenite on JNK activation was not investigated in this study. In this work, a 30 min clamping of the renal pedicles induced only a discrete activation of p-p38 in the $24 \mathrm{~h}$ posthypoxic kidneys from female mice. However, the activation of p-p38 was detected in the $24 \mathrm{~h}$ posthypoxic wild-type RTECs after addition of mineral oil for $30 \mathrm{~min}$. Therefore, the apparent differences observed in the activation of p-p38 may reflect differences in the time course of MAPK activation during IRI, and in cell susceptibility to hypoxia between the in vivo and in vitro experimental conditions. They also may reflect the differences in susceptibility between male and female animals to renal IRI, because kidneys of males exhibit much higher susceptibility to I/R than females, due to the presence of testosterone. ${ }^{37}$

NOX1 has also been shown to cause JNK activation, and the subsequent cell death of cultured mouse, alveolar type-II, epithelial cells. ${ }^{38}$ In contrast, renal IRI leads to a decrease in NOX1, and experiments using Nox1 ${ }^{-1-}$ RTECs clearly show that the extinction of Nox1 mRNA does not affect the activation of $\mathrm{p}-\mathrm{ASK} 1$ and $\mathrm{p}$-JNK in posthypoxic RTECs, suggesting that NOX1- and NOX4-mediated cell apoptosis are tissue specific.

Although TLR4 downstream signaling has an important role in the control of apoptosis during IRI, along with others, we have reported that ischemic kidneys from Tlr2 ${ }^{-1-}$ mice are also better preserved than ischemic wild-type kidneys to almost the same extent as those of $\mathrm{TIr}^{-/-}$mice. ${ }^{8-10}$ However, the TLR2 and TLR4 downstream signaling pathways engaged during IRI are probably different. For example, the activation of ERK1/2, which depends on the dissociation of the gp96/PP5 complex, occurs in posthypoxic wild-type and TIr4 ${ }^{-/-}$RTECs, but not in posthypoxic TIr2 ${ }^{-/}$RTECs. $^{13}$ To conclude, this study has revealed that gp96 and the $28-\mathrm{kDa}$ NOX4 isoform, both of which interact with TLR4, are involved in TLR4-mediated apoptosis in posthypoxic RTECs. The identification of NOX4 as a sensor in the ER stress-induced, TLR4-mediated, pro-apoptotic signaling cascade has important implications for selective anti-superoxide-generating enzyme strategies intended to prevent the apoptosis of ER stress-induced RTECs that may contribute to the deterioration of renal function.

\section{Materials and Methods}

Mice. Pathogen-free, 10- to 12-week-old female wild-type C57BL/6J mice were obtained from the Jackson Laboratory (Bar Harbor, MA, USA). Trr4 ${ }^{-1-}$ and $M y D 88^{-1-}$ null mice (originally obtained from S Akira, Osaka University, Osaka,

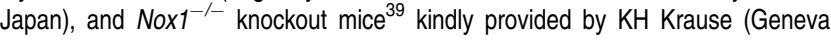
University, Switzerland) were also used. All the mice were further backcrossed at least eight times with $\mathrm{C} 57 \mathrm{BL} / 6 \mathrm{~J}$ mice to ensure that they all had similar genetic backgrounds. All experiments were conducted in accordance with the guidelines of the French Agricultural Office, and in compliance with the legislation governing animal studies.

Renal ischemia/reperfusion in mice. Renal IRI was induced as previously described, but with some slight modifications. ${ }^{7,13}$ Mice were anesthetized by the intraperitoneal injection of 2,2,2-tribromoethanol $(5 \mathrm{mg}$ per mouse) (Sigma-Aldrich, St Louis, MO, USA). The kidneys were exposed by bilateral lumbar incisions, and the renal pedicles were clamped with nontraumatic microvascular clamps for $30 \mathrm{~min}$. The clamps were then removed, and the lumbar incisions were sutured after checking that the blood flow had been restored. Sham-operated mice (referred as controls) underwent the same procedure, except that the renal vessels were not clamped. Surgery was performed on a homeothermic table to maintain a body temperature between 36.5 and $38^{\circ} \mathrm{C}$. All mice received $200 \mu \mathrm{l}$ of sterile $0.9 \% \mathrm{NaCl}$ dripped onto the lumbar incisions to keep the tissue moist. Mice (6-10 mice per group) were killed 1, 2, and 7 days after surgery. Before killing, a blood sample was collected, and the level of serum creatinine was determined using an Olympus AU400 autoanalyzer (Olympus, Hamburg, Germany).

Cultured cells. Experiments were carried out on primary cultured RTECs isolated from the kidneys of naive, wild-type mice, $T / r 4^{-1-}$, or MyD $88^{-1-}$ mice using the same procedure as previously described. ${ }^{13}$ Experiments were also carried out on established mouse PKSV-PR cells exhibiting features of proximal tubule cells. ${ }^{28}$ Hypoxia was induced by immersing RTECs layers in mineral oil as described. ${ }^{7,13}$ After replacing with fresh medium, we added $1 \mathrm{ml}$ sterile mineral oil (Sigma-Aldrich) for $30 \mathrm{~min}$ (for primary cultured RTECs) or $2 \mathrm{~h}$ (for PKSV-PR cells) at $37^{\circ} \mathrm{C}$. The mineral oil was then removed by rinsing twice with fresh medium, and the cells were then incubated at $37^{\circ} \mathrm{C}$ for a further $24 \mathrm{~h}$ before being analyzed. Cells were labeled with an active caspase-3 antibody (Promega, Charbonnières, France) to estimate the percentage of apoptotic cells determined on 2 or 3 enlarged fields $(\times 200)$ from three to five separate cultures derived from two to three different wild-type and/or transgenic mice kidneys for each condition tested. All reagents, media, and buffers used in the assays were checked by using the Limulus amebocyte gelation activity test (BioWhittaker Inc., Walkersville, MD, USA) to ensure that they had not been contaminated by endotoxin.

Immunohistochemical studies. Immunohistochemical studies were performed on deparaffinized kidney sections using antibodies raised against mouse NOX1 and NOX4 or activated caspase-3 (Promega) using avidin-biotin blocking, alkaline phosphatase, and peroxidase substrate kits (Vector Laboratories, Burlingame, CA, USA) according to the manufacturer's instructions. To generate antibodies to NOX1 and NOX4, we used the synthetic peptides ${ }^{332}$ KFQRADNKLKTQKIY $^{346}$ (NOX1) and ${ }^{564}$ NSYGTKFEYNKESFS ${ }^{578}$ (NOX4) as immunogens, and affinity-purified the antisera obtained from immunized rabbits using the corresponding synthetic peptides.

TUNEL. The number of apoptotic cells analyzed in kidney tissue sections fixed in Dubosc-Brazil solution was determined by the TUNEL method using the Apop Tag kit (Chemicon Int., Temecula, CA, USA) according to the manufacturer's instructions. The percentage of apoptotic cells was determined on two or three enlarged fields $(\times 200)$ from two to three different tissue sections from three to five kidneys for each condition tested. ${ }^{13}$

Small-interfering RNA. Experiments were performed using predesigned HP GenomeWide (Qiagen, Courtaboeuf, France) siRNAs for the murine gp96 gene (5'-ATGAATGATATCAAACCAATA-3'; sense, GAAUGAUAUCAAACCAAUAdTdT; antisense, UAUUGGUUUGAUAUCAUUCdAdT), the murine Traf2 gene $\left(5^{\prime}\right.$ ACGGAGTGTCCTGCATGTAAA-3'; sense, GGAGUGUCCUGCAUGUAAATT; antisense, UUUACAUGCAGGACACUCCGT), the murine Nox4 variant $\left(\mathrm{Nox}_{28}\right)$ encoding for only the 28-kDa NOX4 (5'-AATGTTGGGCTGTCCTACTGA-3'; sense, UGUUGGGCUGUCCUACUGAdTdT; antisense, UCAGUAGGACAGCCCAACA $\mathrm{dTdT}$ ). For the murine Nox4 gene encoding both the full-length $65-\mathrm{kDa}$ and the 28-kDa isoforms (Nox465-28), two siRNAs were used to target two sequences $\left(5^{\prime}\right.$ AACGAAGGGGTTAAACACCTC- $3^{\prime}$ and $5^{\prime}$-AAAAGCAAGACTCTACACATC- $3^{\prime}$ ) (Ildiko Szanto, Geneva, Switzerland). A universal negative control siRNA (target DNA sequence, AATTCTCCGAACGTGTCACGT; sense, UUCUCCGAACGU GUCACGUdTdT; antisense, ACGUGACACGUUCGGAGAAdTdT) was also used (Qiagen). Single strand sense and antisense RNA nucleotides were annealed $\left(90^{\circ} \mathrm{C}\right.$ for $1 \mathrm{~min}$, and then $37^{\circ} \mathrm{C}$ for $1 \mathrm{~h}$ ) to generate an RNA duplex according to the 
manufacturer's instructions. Subconfluent cultures of RTECs (day 3-5 after seeding) grown in 48-well plates were incubated with 1-6 pmol of each siRNA tested and $2 \mu$ lof INTERFERin prepared according to the manufacturer's instructions (PolyplusTransfection Inc., San Marcos, CA, USA) for $48 \mathrm{~h}$ at $37^{\circ} \mathrm{C}$ before use.

ROS production assays. The production of ROS produced by intact RTECs was also measured by imaging microscopy. ${ }^{26}$ Untreated and posthypoxic RTECs plated on Lab-Tek chambered coverglass slides (Nalge Nunc International, Roskilde, Denmark) were loaded with $50 \mu \mathrm{M}$ DCFH-DA for $30 \mathrm{~min}$. Cells were then rinsed and after excitation at $488 \mathrm{~nm}$, the green fluorescence of DCF was measured by confocal laser scanning microscopy (CLSM-510-META, Carl Zeiss, Le Pecq, France) equipped with cell culture chamber at $37^{\circ} \mathrm{C}$ and under an atmosphere containing $5 \% \mathrm{CO}_{2}$. When used, DPI $(2 \mu \mathrm{M})$ was added $10 \mathrm{~min}$ before loading the DCFH-DA.

Immunoblot and immunoprecipitation analyses. Frozen kidney samples and cultured RTECs grown in 48-well plates were lysed in $50 \mu \mathrm{l}$ ice-cold lysis buffer containing $62.5 \mathrm{mM}$ Tris- $\mathrm{HCl}(\mathrm{pH} 6.8), 2 \%$ SDS, $10 \%$ glycerol, $50 \mathrm{mM}$ $\mathrm{DTT}$, and then sonicated for $15 \mathrm{~s}$ at $4^{\circ} \mathrm{C}$. For each condition tested, we pooled cell lysates from eight separate wells. Samples (25-50 $\mu \mathrm{g} /$ lane) were electrophoresed using $10 \%$ SDS-PAGE, and analyzed by immunoblot. ${ }^{40}$ Antibodies against TRAF2, p-ASK1, p38, p-p38, p-JNK, p-c-jun, c-jun, MAPKAPK2, p-MAPKAPK2 (Cell Signaling Technology, Danvers, MA, USA), ASK1, JNK (Santa Cruz Biotechnology Inc., Santa Cruz, CA, USA), $\beta$-actin (Sigma-Aldrich), BAX (Upstate Chemicon, Waltham, MA, USA), Bcl-2 (BD Transduction Laboratories, Lexington, KY, USA), and gp96 (Stressgen, Ann Arbor, MI, USA) were used to detect the corresponding antigens. For the immunoprecipitation studies, we lysed, sonicated, centrifuged, and precleared confluent cultures of nonhypoxic or day-1 posthypoxic wild-type or TIr4 ${ }^{-/-}$RTECs with $75 \mu \mathrm{l}$ protein G Sepharose overnight at $4^{\circ} \mathrm{C}$, as described. ${ }^{13}$ Precleared supernatants were then sequentially incubated with antibodies against TLR4 $(5 \mu \mathrm{g} / \mathrm{ml})$, anti-gp96 $(12 \mu \mathrm{g} / \mathrm{ml})$, or ASK1 $(10 \mu \mathrm{g} / \mathrm{ml})$ for $2 \mathrm{~h}$ at $4{ }^{\circ} \mathrm{C}$, and then with $50 \mu \mathrm{l}$ Protein $\mathrm{G}$ Sepharose overnight at $4^{\circ} \mathrm{C}$. Bound proteins were rinsed twice in lysis buffers (Roche Diagnostics), and Sepharose-bound proteins were separated on $8-15 \%$ SDS acrylamide gels (Roche Diagnostics, Mannheim, Germany). After being transferred onto nitrocellulose, we incubated the membranes with antibodies directed against gp96 $(1: 2000)$, TRAF2 $(1: 1000)$, TLR4 $(1: 200)$, and/or NOX4 $(1: 500)$, and then with a peroxidase-conjugated secondary antibody. Labeled bands were detected using the ECL + Western Blotting Detection System (GE Healthcare, Buckinghamshire, UK).

Reverse-transcription PCR. Total RNA was extracted from confluent cultures of RTECs using the RNeasy mini kit according to the manufacturer's instructions, and reverse-transcribed using Moloney Murine Leukemia Virus reverse transcriptase (Invitrogen, Cergy-Pontoise, France). cDNA and non-reversetranscribed RNA (250 ng) from cultured cells were amplified for 35 cycles in $40 \mu \mathrm{l}$ total PCR buffer ( $50 \mathrm{mM} \mathrm{KCl}, 20 \mathrm{mM}$ Tris- $\mathrm{HCl}(\mathrm{pH} 8.4))$ containing $100 \mu \mathrm{M}$ dNTP, 1 or $1.5 \mathrm{mM} \mathrm{MgCl}_{2}, 1 \mathrm{U}$ Taq polymerase, 10 pmol Nox4 ${ }_{65-28}$ (NM 015760.4: nt 43-64, nt 1300-1278), 10 pmol Nox4 28 (NM 015760: nt 43-64, nt 1137-194), or 10 pmol GAPDH (AK 144690: nt 307-327, nt 663-643). The thermal cycling program was $94^{\circ} \mathrm{C}$ for $30 \mathrm{~s}, 56^{\circ} \mathrm{C}$ for $30 \mathrm{~s}$, and then $72^{\circ} \mathrm{C}$ for $1 \mathrm{~min}$. Amplification products were run on a $2 \%$ agarose gel, and then stained with ethidium bromide and autoradiographed.

Statistical analysis. Values are given as means \pm S.E.M. Data are representative of at least three independent experiments. Statistical differences between groups were analyzed using the Student's $t$-test. A $P$-value of $<0.05$ was considered significant.

\section{Conflict of interest}

The authors declare no conflict of interest.

Acknowledgements. We thank S Akira for the use of $T / r 4^{-1-}$ and $M y D 88^{-1-}$ mice. We also thank KH Krause for stimulating discussions, and for providing us with the NOX1 $1^{-1-}$ mice. We thank MW Hornef (Institut of Medical Microbiology and Hygiene, Hannover Medical School, Hannover, Germany) for the gift of the TLR4 antibody. We also thank M Fay and G Pinon (INSERM U773, Paris, France), and B Fernandez and N Quellard (Service d'Anatomie et Cytologie Pathologiques, Hopital
Universitaire de Poitiers, France) for their expert technical assistance. This work was funded by INSERM and in part by an Agence Nationale de la Recherche grant (ANR-08-MIEN-030, to AV). SBM was supported by a PhD student grant from the Ligue Nationale contre le Cancer, AV was in receipt of an Interface INSERM-AP-HP fellowship.

1. Bonventre JV, Weinberg JM. Recent advances in the pathophysiology of ischemic acute renal failure. J Am Soc Nephrol 2003; 14: 2199-2210.

2. Perico N, Cattaneo D, Sayegh MH, Remuzzi G. Delayed graft function in kidney transplantation. Lancet 2004; 364: 1814-1827.

3. Gribar SC, Richardson WM, Sodhi CP, Hackam DJ. No longer an innocent bystander: epithelial toll-like receptor signaling in the development of mucosal inflammation. Mol Med 2008; 14: 645-659.

4. Tsung A, Klune JR, Zhang X, Jeyabalan G, Cao Z, Peng X et al. HMGB1 release induced by liver ischemia involves Toll-like receptor 4 dependent reactive oxygen species production and calcium-mediated signaling. J Exp Med 2007; 204: 2913-2923.

5. Oyama J, Blais Jr C, Liu X, Pu M, Kobzik L, Kelly RA et al. Reduced myocardial ischemia-reperfusion injury in toll-like receptor 4-deficient mice. Circulation 2004; 109: 784-789.

6. Barsness KA, Arcaroli J, Harken AH, Abraham E, Banerjee A, Reznikov L et al. Hemorrhage-induced acute lung injury is TLR-4 dependent. Am J Physiol Regul Integr Comp Physiol 2004; 287: R592-R599.

7. Leemans JC, Stokman G, Claessen N, Rouschop KM, Teske GJ, Kirschning CJ et al. Renal-associated TLR2 mediates ischemia/reperfusion injury in the kidney. J Clin Invest 2005; 115: 2894-2903.

8. Shigeoka AA, Holscher TD, King AJ, Hall FW, Kiosses WB, Tobias PS et al. TLR2 is constitutively expressed within the kidney and participates in ischemic renal injury through both MyD88-dependent and -independent pathways. J Immunol 2007; 178: $6252-6258$.

9. Wu H, Chen G, Wyburn KR, Yin J, Bertolino P, Eris JM et al. TLR4 activation mediates kidney ischemia/reperfusion injury. J Clin Invest 2007; 117: 2847-2859.

10. Wolfs TG, Buurman WA, van Schadewijk A, de Vries B, Daemen MA, Hiemstra PS et al. In vivo expression of Toll-like receptor 2 and 4 by renal epithelial cells: IFN-gamma and TNF-alpha mediated up-regulation during inflammation. J Immunol 2002; 168 : 1286-1293.

11. Kim BS, Lim SW, Li C, Kim JS, Sun BK, Ahn KO et al. Ischemia-reperfusion injury activates innate immunity in rat kidneys. Transplantation 2005; 79: 1370-1377.

12. Yang $Y$, Liu B, Dai J, Srivastava PK, Zammit DJ, Lefrançois $L$ et al. Heat shock protein gp96 is a master chaperone for toll-like receptors and is important in the innate function of macrophages. Immunity 2007; 26: 215-226.

13. Mkaddem SB, Werts C, Goujon JM, Bens M, Pedruzzi E, Ogier-Denis E et al. Heat shock protein gp96 interacts with protein phosphatase 5 and controls toll-like receptor 2 (TLR2)mediated activation of extracellular signal-regulated kinase (ERK) $1 / 2$ in post-hypoxic kidney cells. J Biol Chem 2009; 284: 12541-12549.

14. Hornef MW, Normark BH, Vandewalle A, Normark S. Intracellular recognition of lipopolysaccharide by toll-like receptor 4 in intestinal epithelial cells. J Exp Med 2003; 198 . 1225-1235.

15. Nakano $\mathrm{H}$, Nakajima A, Sakon-Komazawa S, Piao JH, Xue X, Okumura K. Reactive oxygen species mediate crosstalk between NF-kappaB and JNK. Cell Death Differ 2006; 13: $730-737$.

16. Ichijo H, Nishida E, Irie K, ten Dijke P, Saitoh M, Moriguchi T et al. Induction of apoptosis by ASK1, a mammalian MAPKKK that activates SAPK/JNK and p38 signaling pathways. Science 1997; 275: 90-94.

17. Tobiume K, Matsuzawa A, Takahashi T, Nishitoh H, Morita K, Takeda K et al. ASK1 is required for sustained activations of JNK/p38 MAP kinases and apoptosis. EMBO Rep 2001; 2: 222-228.

18. Noguchi T, Takeda K, Matsuzawa A, Saegusa K, Nakano H, Gohda J et al. Recruitment of tumor necrosis factor receptor-associated factor family proteins to apoptosis signal-regulating kinase 1 signalosome is essential for oxidative stress-induced cell death. J Biol Chem 2005; 280: 37033-37040.

19. Matsuzawa A, Saegusa K, Noguchi T, Sadamitsu C, Nishitoh H, Nagai $S$ et al. ROS-dependent activation of the TRAF6-ASK1-p38 pathway is selectively required for TLR4-mediated innate immunity. Nat Immunol 2005; 6: 587-592.

20. Bedard K, Krause KH. The NOX family of ROS-generating NADPH oxidases: physiology and pathophysiology. Physiol Rev 2007; 87: 245-313.

21. Lambeth JD. NOX enzymes and the biology of reactive oxygen. Nat Rev Immunol 2004; 4: 181-189.

22. Geiszt M, Kopp JB, Varnai $P$, Leto TL. Identification of renox, an NAD(P)H oxidase in kidney. Proc Natl Acad Sci USA 2000; 97: 8010-8014.

23. Shiose A, Kuroda J, Tsuruya K, Hirai M, Hirakata $H$, Naito $S$ et al. A novel superoxideproducing NAD(P)H oxidase in kidney. J Biol Chem 2001; 276: 1417-1423.

24. Park HS, Jung HY, Park EY, Kim J, Lee WJ, Bae YS. Cutting edge: direct interaction of TLR4 with NAD(P)H oxidase 4 isozyme is essential for lipopolysaccharide-induced production of reactive oxygen species and activation of NF-kappa B. J Immunol 2004; 173: 3589-3593.

25. Cory S, Adams JM. The Bcl2 family: regulators of the cellular life-or-death switch. Nat Rev Cancer 2002; 2: 647-656. 
26. Pedruzzi E, Guichard C, Ollivier V, Driss F, Fay M, Prunet $C$ et al. NAD $(P) H$ oxidase Nox-4 mediates 7-ketocholesterol-induced endoplasmic reticulum stress and apoptosis in human aortic smooth muscle cells. Mol Cell Biol 2004; 24: 10703-10717.

27. Randow $F$, Seed $B$. Endoplasmic reticulum chaperone gp 96 is required for innate immunity but not cell viability. Nat Cell Biol 2001; 3: 891-896

28. Lacave R, Bens M, Cartier N, Vallet V, Robine S, Pringault E et al. Functional properties of proximal tubule cell lines derived from transgenic mice harboring L-pyruvate kinase-SV40 (T) antigen hybrid gene. J Cell Sci 1993; 104: 705-712.

29. Chamulitrat W, Schmidt R, Tomakidi P, Stremmel W, Chunglok W, Kawahara T et al. Association of gp91phox homolog Nox1 with anchorage-independent growth and MAP kinase-activation of transformed human keratinocytes. Oncogene 2003; 22 6045-6053.

30. Goyal P, Weissmann N, Rose F, Grimminger F, Schäfers HJ, Seeger W et al. Identification of novel Nox4 splice variants with impact on ROS levels in A549 cells. Biochem Biophys Res Commun 2005; 329: 32-39.

31. Serrander L, Cartier L, Bedard K, Banfi B, Lardy B, Plastre O et al. NOX4 activity is determined by mRNA levels and reveals a unique pattern of ROS generation. Biochem J 2007; 406: 105-114.

32. Xu C, Bailly-Maitre B, Reed JC. Endoplasmic reticulum stress: cell life and death decisions. $J$ Clin Invest 2005; 115: 2656-2664.
33. Yang Y, Li Z. Roles of heat shock protein gp96 in the ER quality control: redundant or unique function? Mol Cells 2005; 20: 173-182.

34. Kamata H, Hirata H. Redox regulation of cellular signalling. Cell Signal 1999; 11: 1-14.

35. Martyn KD, Frederick LM, von Loehneysen K, Dinauer MC, Knaus UG. Functional analysis of Nox4 reveals unique characteristics compared to other NADPH oxidases. Cell Signal 2006; 18: 69-82.

36. Wang Y, Ji HX, Zheng JN, Pei DS, Hu SQ, Qiu SL. Protective effect of selenite on renal ischemia/reperfusion injury through inhibiting ASK1-MKK3-p38 signal pathway. Redox Rep 2009; 14: 243-250.

37. Park KM, Kim Jl, Ahn Y, Bonventre AJ, Bonventre JV. Testosterone is responsible for enhanced susceptibility of males to ischemic renal injury. J Biol Chem 2004; 279: 52282-52292.

38. Pantano C, Anathy V, Ranjan P, Heintz NH, Janssen-Heininger YM. Nonphagocytic oxidase 1 causes death in lung epithelial cells via a TNF-RI-JNK signaling axis. Am J Respir Cell Mol Biol 2007; 36: 473-479.

39. Gavazzi G, Banfi B, Deffert C, Fiette L, Schappi M, Herrmann F et al. Decreased blood pressure in NOX1-deficient mice. FEBS Lett 2006; 580: 497-504.

40. Chassin C, Goujon JM, Darche S, du Merle L, Bens M, Cluzeaud F et al. Renal collecting duct epithelial cells react to pyelonephritis-associated Escherichia coli by activating distinct TLR4-dependent and -independent inflammatory pathways. J Immunol 2006; 177: 4773-4784.

Supplementary Information accompanies the paper on Cell Death and Differentiation website (http://www.nature.com/cdd) 